\title{
150 years Langenbeck's Archives-from case-based to evidence-based to personalized surgery
}

\author{
Hans G. Beger
}

Published online: 9 March 2010

(C) Springer-Verlag 2010

Langenbeck's Archive, founded in 1860 in Berlin by B. Langenbeck, Th. Billroth und G. Gurlt, is considered to be, worldwide, the oldest scientific surgical journal being continuously published since 150 years. After introducing in clinical routine, in 1846, ether narcosis and, in 1867, surgical antisepsis in operating theatres and patients' wounds, the application of surgical procedures and the advancement of surgical sciences took fascinating and breathtaking upturns worldwide. Major inventions and clinical inaugurations of surgical procedures furnished proof by German surgeons in the second half of the nineteenth century. Performing a first procedure with application of a new technique was a case-based experience of surgeons. All first surgical procedures were applied after having finished animal experiments to study the technical aspect and to objectify the early consequences of the procedure. W. Kausch, the inaugurator of the KauschWhipple procedure performed hundreds of pancreatic operations in geese, as he was an assistant of the internist Naunyn in Strasbourg. Animal experiments and studies in corpses were considered to be necessary research work to reduce the risk of failure before applying a new surgical treatment to patients. However, all the first surgical procedures applied are based on meticulous observations of one or a few cases before operation and the condition of the individual patient to be operated, but the risk of surgical procedures was still high. Of the nine first distal gastric

H. G. Beger $(\bowtie)$

c/o University Hospital Ulm,

Steinhövelstr. 9,

89075 Ulm, Germany

e-mail: Hans.Beger@uniklinik-ulm.de resections, only two patients survived. Miculicz, assistant in Billroth's Clinic, presented in 1882 the three operative specimens of gastric resections performed by Th. Billroth in Wien, including the first successful long-term survivor applied in 1881 (Arch Klin Chir 1882;11).

To enhance the presentation of cumulative experience of surgical procedures of many cases, the German Society of Surgery initiated during the founding meeting in 1872 a commission for surgical statistics leading to a standardized reporting of diagnosis and surgical procedures.

In the twentieth century, surgical advances were largely made by progress in basic sciences like histology, cell physiology, molecular biology, biochemistry, immunology, and in radiology.

Animal experiments, laboratory research, and standardized observation of series of patients established surgery and surgical science as a major force of progress in medicine in the second half of the twentieth century. However, as surgery advanced, scientific certainty about a final result of the application of two different surgical procedures regarding long-term outcome was difficult to know for certain. The effectivity of lumpectomy compared with modified radical mastectomy was less obvious but of importance for breast cancer patients. The answer to many of these questions is yielded by introducing a control group into the trial. The randomized controlled clinical trial became accepted as an optimal study design for determining treatment effectiveness to exclude differences caused by patient selection, perioperative care, and individual surgical expertise. Randomized controlled trials have today become the accepted method for the evidence of superiority of surgical procedures or drugs.

Performing randomized clinical trials are more difficult in surgery because of blinding, lack of acceptance by patients, and difficulties to standardize surgical procedures. 
Rapid change in technologies, which is particularly observed in use of minimal invasive surgical techniques, and requirement of large samples sizes to detect small differences are additionally rocks at the avenue of progress by randomized, controlled, clinical trials to assess surgical procedures. The prophylactic use of antibiotics in necrotizing pancreatitis has been evaluated by more than eight prospective randomized clinical trials, six of them open trials. Performing a critical meta-analysis, the authors found that the setting was reported incompletely, selection criteria were imprecise reported, and the trials are considered to be underpowered (Pancreas 2007;7:531). Only the two with the highest performance ranking of the eight trials were double blinded, and both of them showed ineffectivity of antibiotic prophylaxis contrary to the results of six randomized controlled trials with regard to frequency of infected necrosis, decrease of mortality, and prevention of surgical intervention. Comparing the effects of randomized controlled clinical trials with observational studies in digestive surgery, Shikata et al. (Ann Surg 2006;244:668) concluded that careful observation of series of patients may lead to correct conclusions about effectiveness of treatment in many instances; they found that only one fourth of the observational studies gave different results from randomized controlled trials.

Breast cancer is a complex disease caused by progressive accumulation of gene mutations combined with epigenetic deregulation of genes and protein pathways. There is growing evidence that known breast cancer risk factors vary by hormone receptor status and possibly other pathologic characteristics of the disease.

Recently, large molecular and cumulative immunological studies resulted in the discovery of novel breast cancer susceptibility loci in genetic and epigenetic regions correlated to heterogenetics by tumor characteristics and the outcome (Clin Cancer Res 2008). A triple negative breast cancer has been found as a subgroup with different outcomes and therapeutic approaches (Clin Cancer Res 2008).

The future of cancer treatment including surgery is going to be based on the individual receptor status and gene activated patterns. Application of personalized multimodal treatment concepts is to be evolving not only in breast cancer but also in colon cancer and cancer of the pancreas and other tumor entities (Langenbeck's Arch Surg 2010;395).

The 150 years of Langenbeck's Archives reflects the evolution of surgery from one case-based experience to evaluation of effectiveness of surgical treatment by controlled clinical trials and recently to a change to personalized medicine to further improve the treatment results.

The advancement of surgery, however, is still today based on what was in 1874 considered to be the primary foundation of surgery:

Permanent fortune in surgery, privileged surgeons, who always have good charts don't exist. Knowledge and skills are the only factors which determine the results (Volkmann, Archiv für Klinische Chirurgie 1874). 